\title{
Epitaxial stabilization of (111)-oriented frustrated quantum pyrochlore thin films
}

\author{
Fangdi Wen, ${ }^{1}$, a) Tsung-Chi Wu, ${ }^{1}$ Xiaoran Liu, ${ }^{1}$ Michael Terilli, ${ }^{1}$ Mikhail Kareev, ${ }^{1}$ and Jak Chakhalian ${ }^{1}$ \\ Department of Physics and Astronomy, Rutgers University, Piscataway, New Jersey 08854, \\ USA
}

(Dated: 2 November 2020)

\begin{abstract}
Frustrated rare-earth pyrochlore titanates, $\mathrm{Yb}_{2} \mathrm{Ti}_{2} \mathrm{O}_{7}$ and $\mathrm{Tb}_{2} \mathrm{Ti}_{2} \mathrm{O}_{7}$, have been proposed as promising candidates to realize quantum spin ice (QSI). Multiple exotic quantum phases, including Coulombic ferromagnet, quantum valence-bond solid, and quadrupolar ordering, have been predicted to emerge in the QSI state upon the application of a (111)-oriented external magnetic field. Here, we report on the primal successful layer-by-layer growth of thin films of frustrated quantum pyrochlores, $\mathrm{R}_{2} \mathrm{Ti}_{2} \mathrm{O}_{7}(\mathrm{R}=\mathrm{Er}, \mathrm{Yb}$, and $\mathrm{Tb})$, along the (111) direction. We confirm their high crystallinity and proper chemical composition by a combination of methods, including in-situ RHEED, x-ray diffraction, reciprocal space mapping, and x-ray photoelectron spectroscopy. The availability of large area (111)-oriented QSI structures with planar geometry offers a new complementary to the bulk platform to explore strain and magnetic field dependent properties in the quasi-2D limit.
\end{abstract}

\section{INTRODUCTION}

In the context of modern condensed matter, there has been a growing interest in searching for exotic states and excitations in highly frustrated materials systems. For example, the excitations of magnetic monopoles due to the fractionalized dipolar degrees of freedom can be found in classical spin ice (CSI) materials such as $\mathrm{Ho}_{2} \mathrm{Ti}_{2} \mathrm{O}_{7}$ and $\mathrm{Dy}_{2} \mathrm{Ti}_{2} \mathrm{O}_{7}$, the rare-earth pyrochlore oxides $1+10$ Another intriguing state of correlated matter is quantum spin liquid (QSL), characterized by emergent fractionalized excitations with long-range entanglement.

Recently, a new class of QSLs named quantum spin ice (QSI) has been theoretically proposed by including quantum fluctuations, which act to prevent the spins from reaching a symmetry-breaking ordered phase of CSIs 9 918-24 QSI has drawn much attention since it supports a QSL ground state described in the framework of compact lattice gauge theory with exotic excitations, including magnetic monopoles, $\mathrm{U}(1)$ gauge photons, and spinons $\frac{1922325 \cdot 30}{-3 n t e r e s t i n g l y, ~ e v e n ~ i f ~ a ~}$ QSI phase is not ultimately realized, due to its highly frustrated nature, proximity to the QSI state is anticipated to induce several unusual quantum phases $\frac{1821 / 2326131+36}{13}$

Experimentally, many frustrated quantum pyrochlores, including $\mathrm{R}_{2} \mathrm{Ti}_{2} \mathrm{O}_{7}(\mathrm{R}=\mathrm{Tb}$ and $\mathrm{Yb})$, have been put forward as promising candidates for QSI.920[29]30]37-46 With the localized nature of $f$-shell moments as well as strong spinorbit interaction, these $f$-electron systems exhibit highly directional exchange interactions akin to those found in $\mathrm{Ki}$ taev magnets, resulting in strongly frustrated behavior $[947+50$ From the experimental viewpoint, however, more often than not, the exact nature of the ground state and low-energy excitations in these quantum pyrochlores remains largely unresolved. One reason is the lack of detailed knowledge on the role of disorder. ${ }^{42[51+54]}$ To illustrate, recently it was found that disorder can promote a new phase in the candidate QSI py-

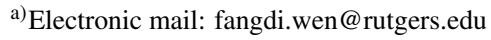

rochlore $\operatorname{Pr}_{2} \mathrm{Zr}_{2} \mathrm{O}_{7}$, which is disordered and yet exhibits shortrange antiferro-quadrupolar correlations and mimics the QSLlike features in neutron scattering. ${ }^{52[55}$ Another challenge is the difficulty of fabricating high-quality single-crystalline samples with low disorders, which hinders the intrinsic QSI physics and unavoidably gives rise to inconsistency between experiments. $\frac{[56] 63}{6}$ To address these challenges, it is thus critical to develop new methods of materials synthesis that are distinct from the conventional solid-state synthesis route to unveil the true nature of frustrated quantum pyrochlores.

One compelling approach that has attracted significant recent interest is to grow the pyrochlore materials as thinfilms oriented along the (111) direction. $\frac{6467}{67}$ The resulting films contain alternating kagome and triangular atomic planes of magnetically active rare-earth ions, which are naturally formed in such orientation and are known to support emer-

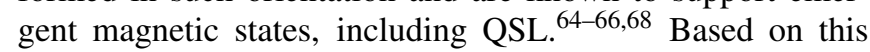
motif, many exotic phenomena including quantum kagome valence bond solid, Coulombic ferromagnet, quadrupolar, and monopole super-solids were proposed to emerge in the QSI films under a magnetic field applied along the (111) direction .6970 Surprisingly, to date, the synthesis of (111) pyrochlore thin films remains very limited and often relies on access to the commercially unavailable pyrochlore substrate $\mathrm{Y}_{2} \mathrm{Ti}_{2} \mathrm{O}_{7}{ }^{\frac{7172}{172}}$ Besides, the epitaxial control of the QSI films can be vital for potential applications in the subfield of quantum information technology.

Here, we report on the primal successful layer-by-layer growth of high-quality thin films of a series of frustrated quantum pyrochlores, $\mathrm{R}_{2} \mathrm{Ti}_{2} \mathrm{O}_{7}(\mathrm{R}=\mathrm{Er}, \mathrm{Tb}$ and $\mathrm{Yb})$, on the $5 \times 5$ $\mathrm{mm}^{2}$ (111) yttria-stabilized $\mathrm{ZrO}_{2}$ (YSZ) substrate. We confirm the high crystallinity of thin films by in-situ reflected high energy electron diffraction (RHEED), $x$-ray measurements including x-ray diffraction (XRD), and reciprocal space map (RSM), and validate that all the films exhibit correct chemical composition by x-ray photoelectron spectroscopy (XPS). Our work offers a complementary to the bulk route to resolve the puzzles in physics of frustrated quantum pyrochlores and contributes to the possible realization of the long-thought QSI state. 

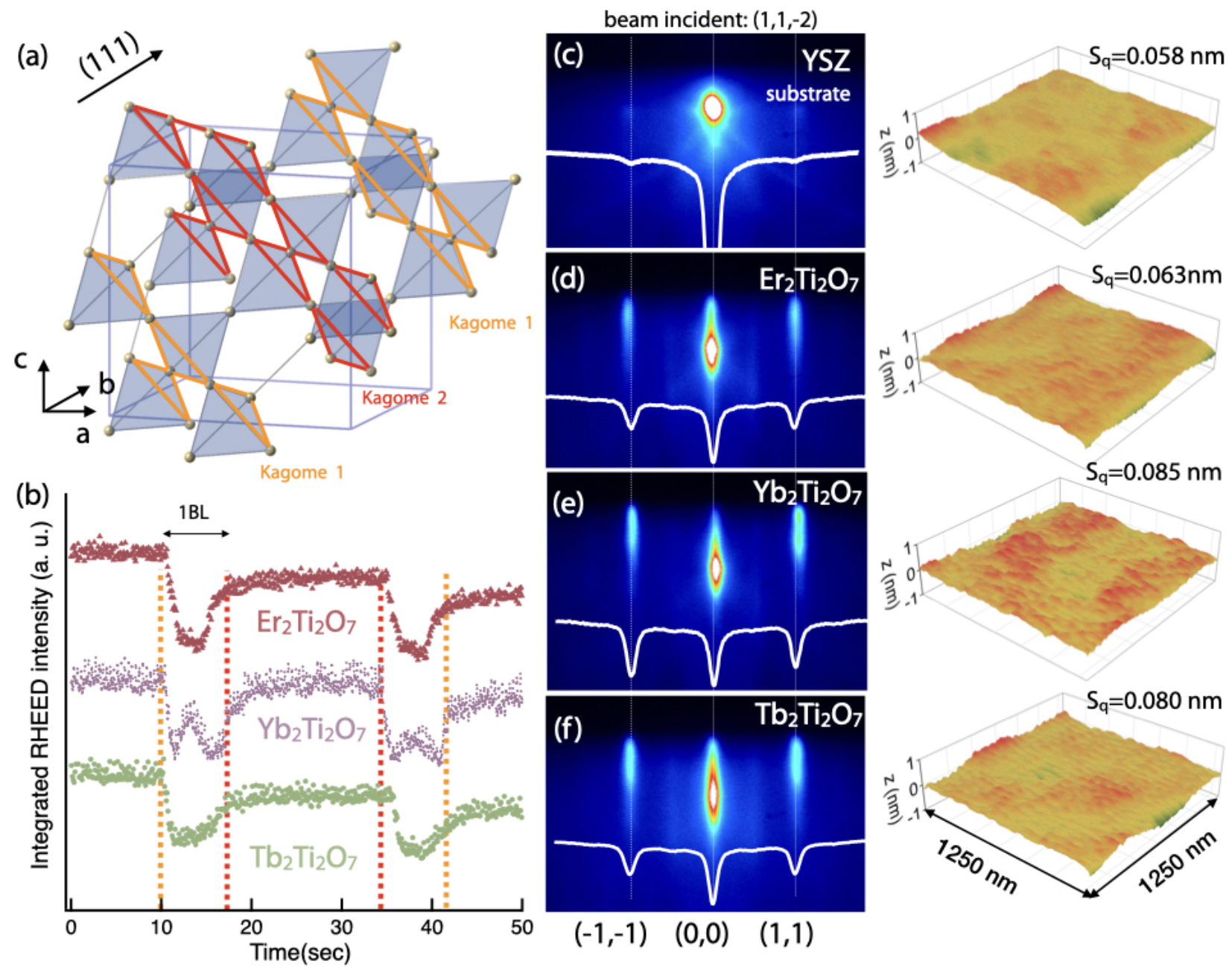

FIG. 1. (a) Schematic picture of $\mathrm{R}$ site sublattice in $\mathrm{R}_{2} \mathrm{Ti}_{2} \mathrm{O}_{7}$. (b) RHEED oscillations during the high-frequency growth, where each interval exhibits the growth of one BL. Note, only the kagome layers are marked for clarity. (c)-(f) RHEED picture of $\mathrm{YSZ}_{2} \mathrm{Er}_{2} \mathrm{Ti}_{2} \mathrm{O}_{7}, \mathrm{Yb}_{2} \mathrm{Ti}_{2} \mathrm{O}_{7}$, and $\mathrm{Tb}_{2} \mathrm{Ti}_{2} \mathrm{O}_{7}$, respectively. Electron beam incident from the $(1,1,-2)$ crystal orientation of YSZ. The white inset curve is a horizontal intensity line cut of the corresponding figure. To the right of (c), (d), (e), and (f) are the AFM of YSZ, $\mathrm{Er}_{2} \mathrm{Ti}_{2} \mathrm{O}_{7}, \mathrm{Yb}_{2} \mathrm{Ti}_{2} \mathrm{O}_{7}$, and $\mathrm{Tb}_{2} \mathrm{Ti}_{2} \mathrm{O}_{7}$ surface. With the same scanning area of $1.25 \mu \mathrm{m} \times 1.25 \mu \mathrm{m}$, the corresponding root mean square roughness obtained by $\mathrm{AFM}$ is: $\mathrm{YSZ}_{-}-58 \mathrm{pm}, \mathrm{Er}_{2} \mathrm{Ti}_{2} \mathrm{O}_{7}-$ $63 \mathrm{pm}, \mathrm{Yb}_{2} \mathrm{Ti}_{2} \mathrm{O}_{7}-85 \mathrm{pm}$, and $\mathrm{Tb}_{2} \mathrm{Ti}_{2} \mathrm{O}_{7}-80 \mathrm{pm}$.

\section{RESULTS AND DISCUSSION}

Three frustrated quantum pyrochlores, $\mathrm{R}_{2} \mathrm{Ti}_{2} \mathrm{O}_{7}(\mathrm{R}=\mathrm{Er}, \mathrm{Tb}$, and $\mathrm{Yb}$ ), were epitaxially stabilized on YSZ substrates using pulsed-laser deposition with in-situ RHEED control at the identical growth condition. Specifically, before the growth, substrates were heated up to $750{ }^{\circ} \mathrm{C}$ at $10{ }^{\circ} \mathrm{C} / \mathrm{min}$ under 120 mTorr oxygen pressure. During the growth, the presence of the layer-by-layer growth mode was determined by highpressure RHEED. All the reported films were deposited at the $18 \mathrm{~Hz}$ repetition rate interrupted by 15 -second intervals. A high-frequency deposition enlarges the supersaturation limit, lowers the nucleation barrier, and thus markedly improves the growth ${ }^{73}$ After the growth, samples were kept at the growth condition for $10 \mathrm{mins}$, and then cooled down to room temperature with the ramp rate of $15^{\circ} \mathrm{C} / \mathrm{min}$. It is noteworthy that no annealing was required in this process.

Surprisingly, as shown in Fig. 1 b), unlike a conventional RHEED pattern expected for interrupted growth, two oscillations were found to appear for all samples albeit with a different number of pulses required to complete a cycle. This unexpected result can be readily explained by the fact that since the rare-earth sites and $\mathrm{Ti}$ sites have the same sublattice structure (but with a small displacement), a kagome layer is composed of rare-earth ions which are coplanar with a triangle layer of $\mathrm{Ti}^{4+}$ ions. Therefore, a complete structural unit requires two inequivalent bilayers $(\mathrm{BL})$ composed of one rareearth kagome plane $\left(\mathrm{K}_{R}\right)$ plus one triangle Ti ions plane $\left(\mathrm{T}_{T}\right)$ or $\mathrm{BL}=\mathrm{K}_{R}-\mathrm{T}_{T}$ and another bilayer composed of kagome $\mathrm{Ti}$ $\left(\mathrm{K}_{T}\right)$ and triangle rare-earth $\left(\mathrm{T}_{R}\right)$ planes, thus overall 1 u.c.= $\mathrm{K}_{R}-\mathrm{T}_{T}-\mathrm{K}_{T}-\mathrm{T}_{R}$. As illustrated in Fig. 1(a), these BLs are not precisely on the top of each other but rather stacked with a 

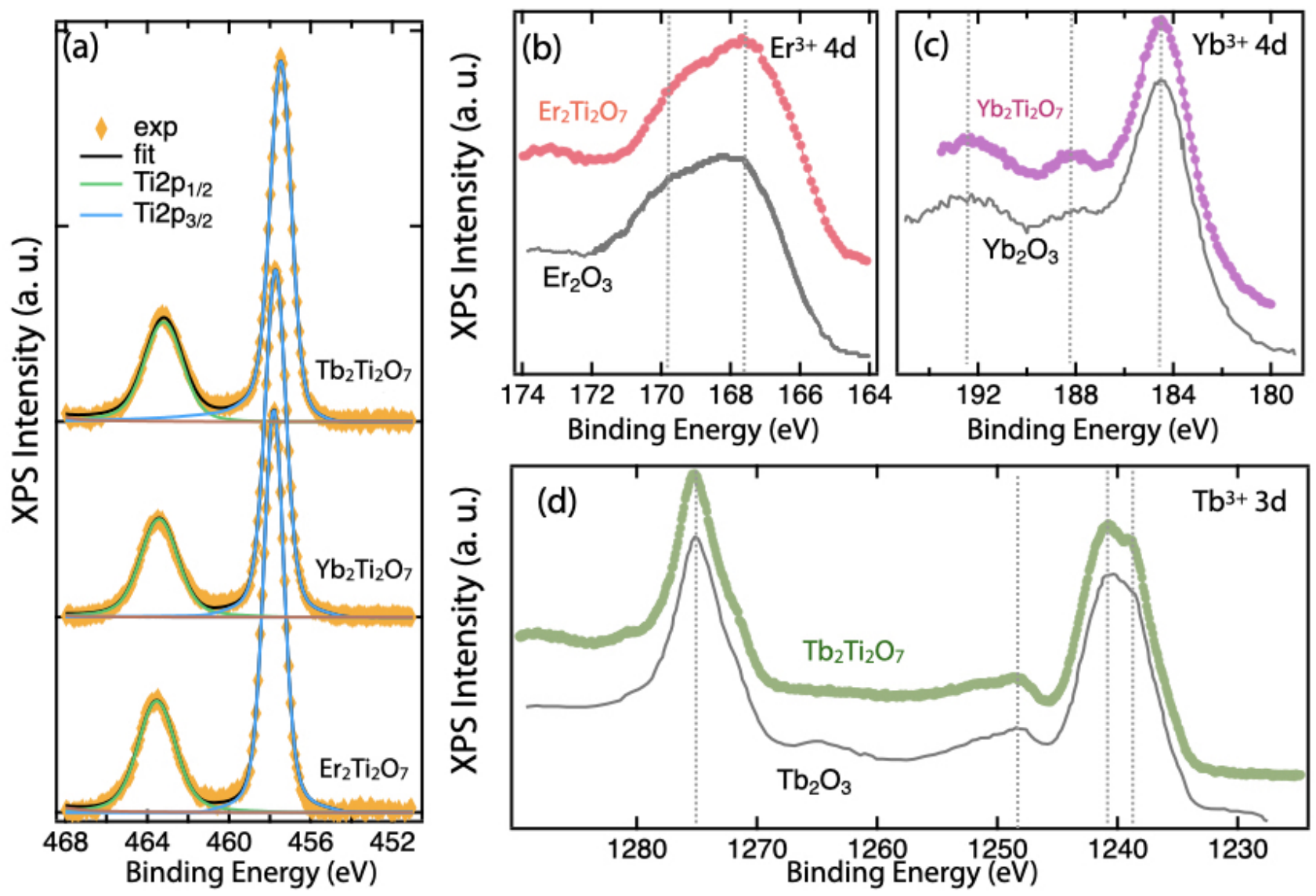

FIG. 2. (a) XPS of Ti $2 p$ on $\mathrm{Er}_{2} \mathrm{Ti}_{2} \mathrm{O}_{7}, \mathrm{Yb}_{2} \mathrm{Ti}_{2} \mathrm{O}_{7}$ and $\mathrm{Tb}_{2} \mathrm{Ti}_{2} \mathrm{O}_{7}$ films. All the background has been subtracted for better comparison. (b) XPS of $\mathrm{Er} 4 \mathrm{~d}$ in $\mathrm{Er}_{2} \mathrm{Ti}_{2} \mathrm{O}_{7}$ sample in comparison to the data adapted from the reported $\mathrm{Er}_{2} \mathrm{O}_{3}{ }^{74}$ (c) XPS of $\mathrm{Yb}_{4} \mathrm{~d}_{\text {in }} \mathrm{Yb}_{2} \mathrm{Ti}_{2} \mathrm{O}_{7}$ sample in comparison to the data adapted from the reported $\mathrm{Yb}_{2} \mathrm{O}_{3} \cdot{ }^{[75}$ (d) XPS of Tb $3 \mathrm{~d}_{\text {in }} \mathrm{Tb}_{2} \mathrm{Ti}_{2} \mathrm{O}_{7}$ sample in comparison to the data adapted from the reported $\mathrm{Tb}_{2} \mathrm{O}_{3}$

small offset . Further thickness analysis revealed that between each interval, we indeed deposited two BLs of material. Furthermore, this structural consideration is in good agreement with the observed doublet structure of RHEED intensity in all (111) oriented films. The ability to see the two-peak structure in RHEED oscillations during high-frequency deposition provides strong evidence for the layer-by-layer growth.

After each deposition, RHEED intensity recovers to almost the same intensity, with a minor decay corresponding to the natural decay of the $\mathrm{LaB}_{6}$ electron source. This result implies that the surface roughness remained roughly constant for each unit cell. The RHEED patterns of all samples, including the YSZ substrate, are shown in the left part of Fig. 11(c)-(f). Because of the small incident angle, the $(-1,-1)$ and $(1,1)$ crystal peaks of YSZ were barely observed. However, soon after the initial deposition, two evident streaky peaks emerge at the ($1,-1)$ and $(1,1)$ positions. A clear half-order reflections can be seen after the deposition, which is as expected since the pyrochlore lattice constant is twice of that of YSZ. When cooled down to $300^{\circ} \mathrm{C}$, the RHEED pattern remained unchanged and disappeared at the room temperature due to the strong charg- ing effect.

After the growth, each sample was characterized in four aspects: the surface morphology, crystallinity, chemical composition, and crystal structure. Other than determining the surface morphology by in-situ RHEED, atomic force microscopy (AFM) is another commonly-used probe. As seen in the right half of Fig. 1 (c), the YSZ substrate has a root mean square surface roughness $\mathrm{S}_{q}$ of around $58 \mathrm{pm}$ over a $1.25 \mu \mathrm{m} \times 1.25$ $\mu \mathrm{m}$ area. The $\mathrm{S}_{q}$ of $\mathrm{Er}_{2} \mathrm{Ti}_{2} \mathrm{O}_{7}(\mathrm{~d}), \mathrm{Yb}_{2} \mathrm{Ti}_{2} \mathrm{O}_{7}$ (e) and $\mathrm{Tb}_{2} \mathrm{Ti}_{2} \mathrm{O}_{7}$ (f) are all of the same order of 60-80 pm. Remarkably, since each BL corresponds to $80 \mathrm{pm}$, the surface roughness of all three films is within $1 \mathrm{BL}$, which is another manifestation of the atomically-flat surface due to the layer-by-layer growth.

To assure that during the deposition, ions are stabilized in the proper charge state, a detailed XPS analysis has been carried out on both $\mathrm{R}$ (Er, $\mathrm{Yb}$, and $\mathrm{Tb}$ ) and Ti core-states. During the XPS analysis, all the recorded data were calibrated to the carbon $1 s$ peak at $284.5 \mathrm{eV}$ to eliminate the possible charging effect. The XPS core level data of $\mathrm{Ti}^{4+}$ in $\mathrm{Er}_{2} \mathrm{Ti}_{2} \mathrm{O}_{7}$, $\mathrm{Yb}_{2} \mathrm{Ti}_{2} \mathrm{O}_{7}$ and $\mathrm{Tb}_{2} \mathrm{Ti}_{2} \mathrm{O}_{7}$ are shown in Fig.2 (a). For better comparison the Shirley-type background was subtracted from 

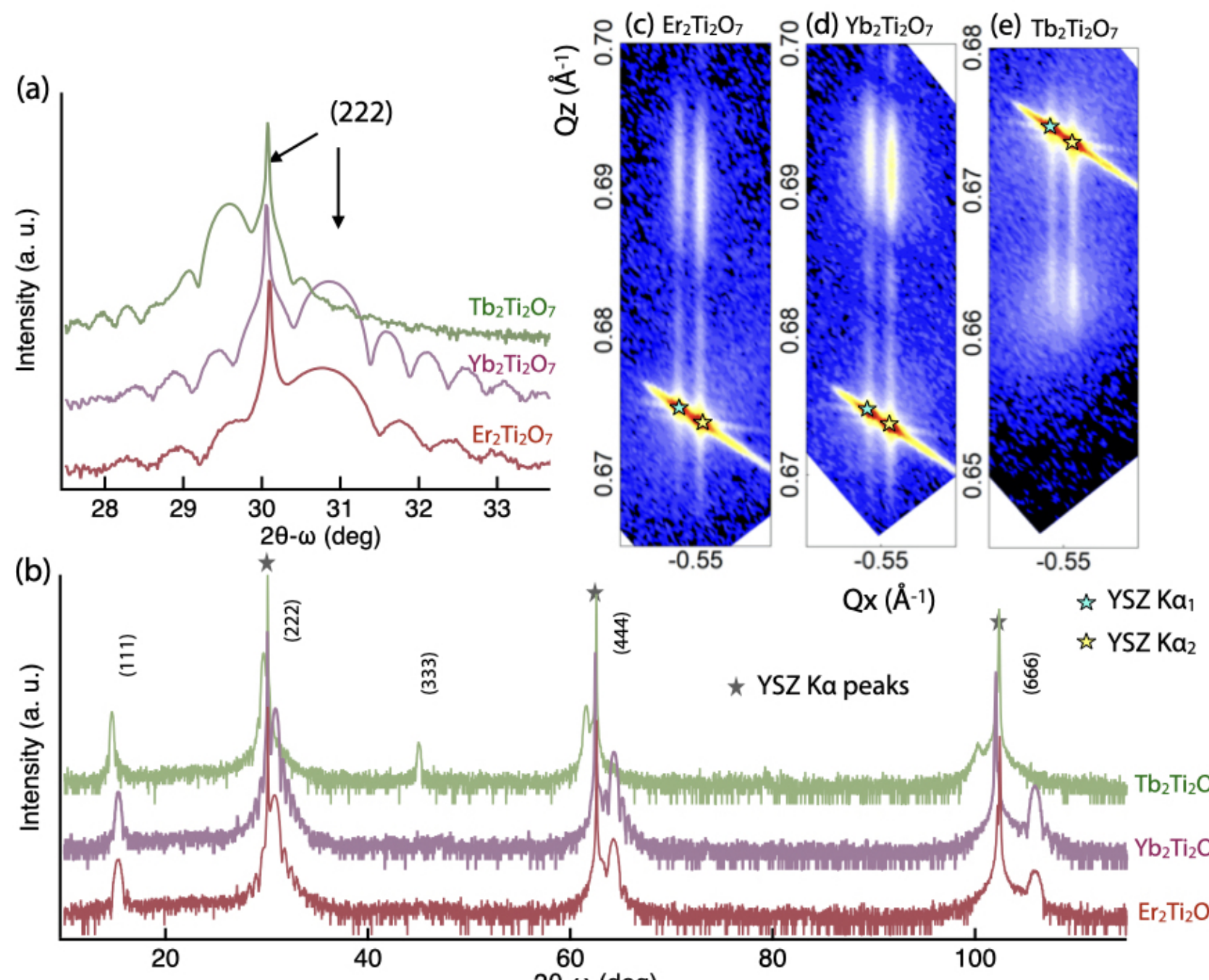

FIG. 3. (a) $\mathrm{XRD}$ of $\mathrm{Er}_{2} \mathrm{Ti}_{2} \mathrm{O}_{7}, \mathrm{Yb}_{2} \mathrm{Ti}_{2} \mathrm{O}_{7}$ and $\mathrm{Tb}_{2} \mathrm{Ti}_{2} \mathrm{O}_{7}$ films near the film peak (222) and substrate peak (111). (b) Full range $\mathrm{XRD}$ of $\mathrm{Er}_{2} \mathrm{Ti}_{2} \mathrm{O}_{7}, \mathrm{Yb}_{2} \mathrm{Ti}_{2} \mathrm{O}_{7}$ and $\mathrm{Tb}_{2} \mathrm{Ti}_{2} \mathrm{O}_{7}$. (c), (d), and (e) are the reciprocal space map of $\mathrm{Er}_{2} \mathrm{Ti}_{2} \mathrm{O}_{7}, \mathrm{Yb}_{2} \mathrm{Ti}_{2} \mathrm{O}_{7}$ and $\mathrm{Tb}_{2} \mathrm{Ti}_{2} \mathrm{O}_{7}$ around the substrate peak $(0,4,2)$ and film peak $(0,8,4)$. Both $\mathrm{K} \alpha_{1}$ and $\mathrm{K} \alpha_{2}$ were detected, and we calculated $\mathrm{Q}$ vectors based on $\mathrm{K} \alpha_{1}$ with wavelength of $(1.5406 \AA)$. All graphs show data acquired from three samples: $\mathrm{Er}_{2} \mathrm{Ti}_{2} \mathrm{O}_{7}$ sample with thickness $16 \mathrm{~nm}, \mathrm{Yb}_{2} \mathrm{Ti}_{2} \mathrm{O}_{7}$ sample with thickness 20nm, and $\mathrm{Tb}_{2} \mathrm{Ti}_{2} \mathrm{O}_{7}$ sample with thickness $30 \mathrm{~nm}$.

all Ti XPS spectra. Ti $2 p_{3 / 2}$ peak and Ti $2 p_{1 / 2}$ peak were fitted with a fixed area ratio of 2:1. The measured binding energy for Ti $2 p_{3 / 2}$ peak is around $458.8 \mathrm{eV}$, which is in good agreement with the reference $\mathrm{Ti}^{4+}$ systems. ${ }^{[7778}$ Although titanium can easily lower its valency, the spectra show no sign of $\mathrm{Ti}^{3+}$ $(\sim 455 \mathrm{eV})$ present in any of the films.

On the other hand, being in the group of lanthanides, Er, Tb, and $\mathrm{Yb}$ almost exclusively form compounds with an oxidation state of +3 . Unlike the relatively simple lineshape of $\mathrm{Ti}^{4+}$ XPS, the XPS spectra of R sites (Er, Yb, Tb) are significantly more complex. To overcome this issue, we compare our results to the reference oxide systems with well-defined charge states. First, as shown in Fig 2(b), though the peak positions of $\mathrm{Er}^{3+}$ vary in different materials systems, the XPS spectrum of $\mathrm{Er}_{2} \mathrm{O}_{3}$ shows a clear correspondence to our data ${ }^{79}$ The observed fine structure of $\mathrm{Er}^{3+}$ can be attributed to the hybridization of Eu ions into Er-O, Er-Er, and Er-Ti bonds and surface contamination. ${ }^{81}$ For $\mathrm{Yb}_{2} \mathrm{Ti}_{2} \mathrm{O}_{7}$, the single $4 d_{5 / 2}$ peak can be clearly seen at around $185 \mathrm{eV}$, which is in good agreement with previous XPS result of $\mathrm{Yb}^{3+}$ in $\mathrm{Yb}_{2} \mathrm{O}_{3}$ [see Fig. 2 (c)] ${ }^{82-84} \mathrm{It}$ is worthy to note that if there is $\mathrm{Yb}^{2+}$ present in the material, an extra peak will show at around $181 \mathrm{eV}$, which is not the case for our result. Finally, the spectrum of $\mathrm{Tb}^{3+}$ $3 d$ spectrum is consistent with the XPS spectra reported for $\mathrm{Tb}_{2} \mathrm{O}_{3}{ }^{8566}$ At this point we can conclude that in all synthesised films show correct $\mathrm{R}^{3+}$ (i.e. $\mathrm{Er}^{3+}, \mathrm{Yb}^{3+}, \mathrm{Tb}^{3+}$ ) and $\mathrm{Ti}^{4+}$ oxidation states.

Next, we discuss the structural properties of the films. 
In the bulk, the reported lattice constants for $\mathrm{Er}_{2} \mathrm{Ti}_{2} \mathrm{O}_{7}$ and $\mathrm{Yb}_{2} \mathrm{Ti}_{2} \mathrm{O}_{7}$ are $10.075 \AA$ and $10.032 \AA$, respectively ${ }^{87}[88$ Their lattice constants correspond to the spacing of $5.82 \AA$ for $\mathrm{Er}_{2} \mathrm{Ti}_{2} \mathrm{O}_{7}$ and $5.79 \AA$ for $\mathrm{Yb}_{2} \mathrm{Ti}_{2} \mathrm{O}_{7}$ for each BL grown along the (111) direction. However, from the bulk result, it is known that the lattice constant of $\mathrm{Tb}_{2} \mathrm{Ti}_{2} \mathrm{O}_{7}$ is very sensitive to the growth condition; specifically, depending on the synthesis technique, the lattice constant can vary from 10.127 $\AA^{89}$ to above $10.15 \AA .56757900$ As clearly seen in Fig. 3 (a), $\mathrm{Er}_{2} \mathrm{Ti}_{2} \mathrm{O}_{7}$ and $\mathrm{Yb}_{2} \mathrm{Ti}_{2} \mathrm{O}_{7}$ shows film peaks at higher angles than substrate peaks. The calculated spacing along the (111) direction is estimated to be $5.80 \AA$ for $\mathrm{Er}_{2} \mathrm{Ti}_{2} \mathrm{O}_{7}$ and $5.78 \AA$ for $\mathrm{Yb}_{2} \mathrm{Ti}_{2} \mathrm{O}_{7}$. The smaller out-of-plane lattice spacing indicates tensile strain in the film, which is expected as pyrochlores have the lattice constants smaller than that of the YSZ substrate $(5.12 \AA)$. Also, because $\mathrm{Yb}_{2} \mathrm{Ti}_{2} \mathrm{O}_{7}$ has a more pronounced lattice mismatch with YSZ, it has a smaller outof-plane spacing, which is confirmed by our XRD result.

Notably, the (333) and (555) peaks are not observed in the $\mathrm{Er}_{2} \mathrm{Ti}_{2} \mathrm{O}_{7}$ and $\mathrm{Yb}_{2} \mathrm{Ti}_{2} \mathrm{O}_{7}$ films. If we define the peak ratio between (111) peak and (333) peak as $r$, under cubic symmetry $\mathrm{Fd} \overline{3} \mathrm{~m}, r_{\mathrm{ETO}}=99.56$ and $r_{\mathrm{YTO}}=75.17$, implying that the intensity of the (333) peak is indeed very low compared to the (111) peak. Moreover, even for the bulk crystals of $\mathrm{Er}_{2} \mathrm{Ti}_{2} \mathrm{O}_{7}$ and $\mathrm{Yb}_{2} \mathrm{Ti}_{2} \mathrm{O}_{7}$, the (333) peak is barely observed in neutron or x-ray scattering measurements ${ }^{91-93}$ On the other hand, our XRD measurements on $\mathrm{Tb}_{2} \mathrm{Ti}_{2} \mathrm{O}_{7}$ have revealed several variations in the structural properties, including the lattice constant and intensity of the (333) peak. Here, assuming the $\mathrm{Tb}_{2} \mathrm{Ti}_{2} \mathrm{O}_{7}$ is under Fd $\overline{3} \mathrm{~m}$ space group, and $a_{\mathrm{TTO}}=10.15 \AA$, the estimated $r_{\mathrm{TTO}}=96$. However, our XRD result indicates that the value of $r_{\mathrm{TTO}}^{\exp }=10.44$, which is much smaller than expected for the $\mathrm{Fd} \overline{3} \mathrm{~m}$ space group. In addition, the estimated out-of-plane lattice spacing from (111), (222), (333) and (444) film peaks consistently yeilds $d_{111}=6.03 \AA$, which is by $2.6 \%$ larger than $a_{\text {bulk }}=10.15 \AA\left(d_{111}=5.86 \AA\right)$. Based on the reciprocal space mapping (RSM) [see Fig. 3(c)], the film appear to be fully strained in-plane, therefore the experimental unit cell volume of $\mathrm{Tb}_{2} \mathrm{Ti}_{2} \mathrm{O}_{7}$ is $1095 \AA^{3}$, which is $4.7 \%$ larger than that of the bulk unit cell $\left(1045.7 \AA^{3}\right)$. Despite the observed deviation in the lattice constant of $\mathrm{Tb}_{2} \mathrm{Ti}_{2} \mathrm{O}_{7}$, the XRD scans show no extra peaks other than those of the (111) - oriented crystal, confirming the absence of a secondary chemical phase or domain separation in the $\mathrm{Tb}_{2} \mathrm{Ti}_{2} \mathrm{O}_{7}$ films.

As shown in Fig 3(a)-(b), all films demonstrate clear Kiessig fringes near the film peaks, which result from the interference of the x-ray beams reflected on the film surface and the interface between the film and the substrate. These fringes indicate that our films have high thickness homogeneity and small surface/interface roughness constant with the AFM data. With angle-dependent Kiessig fringes, the film thickness is estimated to be $16 \mathrm{~nm}$ for $\mathrm{Er}_{2} \mathrm{Ti}_{2} \mathrm{O}_{7}, 20 \mathrm{~nm}$ for $\mathrm{Yb}_{2} \mathrm{Ti}_{2} \mathrm{O}_{7}$, and $30 \mathrm{~nm}$ for $\mathrm{Tb}_{2} \mathrm{Ti}_{2} \mathrm{O}_{7}$. Furthermore, the RSM shown in Fig. 3 (c) implies a perfect alignment between the substrate (042) and film peaks (084) horizontally, signifying that all samples are fully-strained. The RSM data also corroborate that the out-of-plane lattice constants of $\mathrm{Er}_{2} \mathrm{Ti}_{2} \mathrm{O}_{7}$ and $\mathrm{Yb}_{2} \mathrm{Ti}_{2} \mathrm{O}_{7}$ are larger than the out-of-plane lattice constant of
YSZ substrate, and smaller for $\mathrm{Tb}_{2} \mathrm{Ti}_{2} \mathrm{O}_{7}$. Altogether, we observed a unique crystal structure in $\mathrm{Tb}_{2} \mathrm{Ti}_{2} \mathrm{O}_{7}$ film that deviates from the commonly-observed in the bulk $\mathrm{Fd} \overline{3} \mathrm{~m}$ cubic structure. The origin of the enlarged unit-cell volume and how the change in the crystal structure may affect its physical properties remain an open question to be studied in the future.

\section{CONCLUSION}

In conclusion, we have epitaxially grown new high-quality frustrated quantum pyrochlores $\mathrm{R}_{2} \mathrm{Ti}_{2} \mathrm{O}_{7}(\mathrm{R}=\mathrm{Er}$, $\mathrm{Tb}$, and $\mathrm{Yb}$ ) on (111)-oriented YSZ substrates in the layer-by-layer mode. All systems exhibit correct chemical composition on both rare-earth sites and Ti site. Structurally, all films are fully strained with high homogeneity and very small surface roughness. Among the three systems, $\mathrm{Er}_{2} \mathrm{Ti}_{2} \mathrm{O}_{7}$ and $\mathrm{Yb}_{2} \mathrm{Ti}_{2} \mathrm{O}_{7}$ demonstrate tensile lattice strain as expected in the assumption of tetragonal distortion, while $\mathrm{Tb}_{2} \mathrm{Ti}_{2} \mathrm{O}_{7}$ shows an unexpectedly larger lattice parameter for the $\mathrm{Fd} \overline{3} \mathrm{~m}$ space group. Our work not only lays a solid ground for highly frustrated lattices grown from pyrochlore materials into thin-film forms in a (111) direction, but also offers a novel materials platform to explore the effects of strain and magnetic field in the quasi2D limit with the unique potential for the possible realization of QSI.

\section{ACKNOWLEDGEMENT AND DATA AVAILABILITY STATEMENTS}

This work was supported by the Gordon and Betty Moore Foundation EPiQS Initiative through Grant No. GBMF4534. The data that support the findings of this study are available from the corresponding author upon reasonable request.

${ }^{1}$ T. Fennell, P. Deen, A. Wildes, K. Schmalzl, D. Prabhakaran, A. Boothroyd, R. Aldus, D. McMorrow, and S. Bramwell, "Magnetic coulomb phase in the spin ice $\mathrm{Ho}_{2} \mathrm{Ti}_{2} \mathrm{O}_{7}$," Science 326, 415-417 (2009).

${ }^{2}$ C. Castelnovo, R. Moessner, and S. L. Sondhi, "Magnetic monopoles in spin ice," Nature 451, 42-45 (2008).

${ }^{3}$ L. D. Jaubert and P. C. Holdsworth, "Signature of magnetic monopole and dirac string dynamics in spin ice," Nat. Phys. 5, 258-261 (2009).

${ }^{4}$ S. T. Bramwell, S. Giblin, S. Calder, R. Aldus, D. Prabhakaran, and T. Fennell, "Measurement of the charge and current of magnetic monopoles in spin ice," Nature 461, 956-959 (2009).

${ }^{5}$ M. J. Harris, S. Bramwell, D. McMorrow, T. Zeiske, and K. Godfrey, "Geometrical frustration in the ferromagnetic pyrochlore $\mathrm{Ho}_{2} \mathrm{Ti}_{2} \mathrm{O}_{7}$," Phys. Rev. Lett. 79, 2554 (1997).

${ }^{6}$ B. C. den Hertog and M. J. Gingras, "Dipolar interactions and origin of spin ice in ising pyrochlore magnets," Phys. Rev. Lett. 84, 3430 (2000).

${ }^{7}$ S. T. Bramwell and M. J. Gingras, "Spin ice state in frustrated magnetic pyrochlore materials," Science 294, 1495-1501 (2001).

${ }^{8}$ J. S. Gardner, M. J. Gingras, and J. E. Greedan, "Magnetic pyrochlore oxides," Rev. Mod. Phys. 82, 53 (2010).

${ }^{9}$ J. G. Rau and M. J. Gingras, "Frustrated quantum rare-earth pyrochlores," Annu. Rev. Condens. Matter Phys. (2019).

${ }^{10}$ C. Castelnovo, R. Moessner, and S. L. Sondhi, "Spin ice, fractionalization, and topological order," Annu. Rev. Condens. Matter Phys. 3, 35-55 (2012).

${ }^{11}$ L. Balents, "Spin liquids in frustrated magnets," Nature 464, 199-208 (2010). 
${ }^{12}$ C. Broholm, R. Cava, S. Kivelson, D. Nocera, M. Norman, and T. Senthil, "Quantum spin liquids," Science 367 (2020).

${ }^{13}$ L. Savary and L. Balents, "Quantum spin liquids: a review," Rep. Prog. Phys. 80, 016502 (2016).

${ }^{14}$ Y. Zhou, K. Kanoda, and T.-K. Ng, "Quantum spin liquid states," Rev. Mod. Phys. 89, 025003 (2017).

${ }^{15}$ J. Knolle and R. Moessner, "A field guide to spin liquids," Annu. Rev. Condens. Matter Phys. 10, 451-472 (2019).

${ }^{16}$ T.-H. Han, J. S. Helton, S. Chu, D. G. Nocera, J. A. Rodriguez-Rivera, C. Broholm, and Y. S. Lee, "Fractionalized excitations in the spin-liquid state of a Kagome-lattice antiferromagnet," Nature 492, 406-410 (2012).

${ }^{17}$ N. Janša, A. Zorko, M. Gomilšek, M. Pregelj, K. W. Krämer, D. Biner, A. Biffin, C. Rüegg, and M. Klanjšek, "Observation of two types of fractional excitation in the Kitaev honeycomb magnet," Nat. Phys. 14, 786-790 (2018).

${ }^{18}$ M. J. Gingras and P. A. McClarty, "Quantum spin ice: a search for gapless quantum spin liquids in pyrochlore magnets,” Rep. Prog. Phys. 77, 056501 (2014).

${ }^{19}$ M. Hermele, M. P. Fisher, and L. Balents, "Pyrochlore photons: The U(1) spin liquid in a $S=1 / 2$ three-dimensional frustrated magnet," Phys. Rev. B 69, 064404 (2004).

${ }^{20}$ K. A. Ross, L. Savary, B. D. Gaulin, and L. Balents, "Quantum excitations in quantum spin ice," Phys. Rev. X 1, 021002 (2011).

${ }^{21}$ S. Lee, S. Onoda, and L. Balents, "Generic quantum spin ice," Phys. Rev. B 86, 104412 (2012).

${ }^{22}$ O. Benton, O. Sikora, and N. Shannon, "Seeing the light: experimental signatures of emergent electromagnetism in a quantum spin ice," Phys. Rev. B 86, 075154 (2012).

${ }^{23}$ L. Savary and L. Balents, "Coulombic quantum liquids in spin-1/2 pyrochlores," Phys. Rev. Lett. 108, 037202 (2012).

${ }^{24}$ M. R. Norman, "Colloquium: Herbertsmithite and the search for the quantum spin liquid," Rev. Mod. Phys. 88, 041002 (2016)

${ }^{25} \mathrm{G}$. Chen, "Dirac's "magnetic monopoles" in pyrochlore ice U(1) spin liquids: Spectrum and classification," Phys. Rev. B 96, 195127 (2017).

${ }^{26}$ B. Gao, T. Chen, D. W. Tam, C.-L. Huang, K. Sasmal, D. T. Adroja, F. Ye, H. Cao, G. Sala, M. B. Stone, C. Baines, J. A. T. Verezhak, H. Hu, J.-H. Chung, X. Xu, S.-W. Cheong, M. Nallaiyan, S. Spagna, M. B. Maple, A. H. Nevidomskyy, E. Morosan, G. Chen, and P. Dai, "Experimental signatures of a three-dimensional quantum spin liquid in effective spin-1/2 $\mathrm{Ce}_{2} \mathrm{Zr}_{2} \mathrm{O}_{7}$ pyrochlore," Nat. Phys. 15, 1052-1057 (2019)

${ }^{27}$ X.-P. Yao, Y.-D. Li, and G. Chen, "Pyrochlore u(1) spin liquid of mixedsymmetry enrichments in magnetic fields," Phys. Rev. Research 2, 013334 (2020)

${ }^{28}$ B. Canals and C. Lacroix, "Pyrochlore antiferromagnet: A threedimensional quantum spin liquid," Phys. Rev. Lett. 80, 2933-2936 (1998)

${ }^{29}$ R. Sibille, E. Lhotel, V. Pomjakushin, C. Baines, T. Fennell, and M. Kenzelmann, "Candidate quantum spin liquid in the $\mathrm{Ce}^{3+}$ Pyrochlore Stannate $\mathrm{Ce}_{2} \mathrm{Sn}_{2} \mathrm{O}_{7}$," Phys. Rev. Lett. 115, 097202 (2015)

${ }^{30}$ L. Clark, G. J. Nilsen, E. Kermarrec, G. Ehlers, K. S. Knight, A. Harrison, J. P. Attfield, and B. D. Gaulin, "From spin glass to quantum spin liquid ground states in molybdate pyrochlores," Phys. Rev. Lett. 113, 117201 (2014)

${ }^{31}$ Y. Wan, J. Carrasquilla, and R. G. Melko, "Spinon walk in quantum spin ice," Phys. Rev. Lett. 116, 167202 (2016).

${ }^{32}$ Y. Kato and S. Onoda, "Numerical evidence of quantum melting of spin ice: quantum-to-classical crossover," Phys. Rev. Lett. 115, 077202 (2015).

${ }^{33}$ O. Benton, L. D. Jaubert, R. R. Singh, J. Oitmaa, and N. Shannon, "Quantum spin ice with frustrated transverse exchange: From a $\pi$-flux phase to a nematic quantum spin liquid," Phys. Rev. Lett. 121, 067201 (2018).

${ }^{34}$ M. Udagawa and R. Moessner, "Spectrum of itinerant fractional excitations in quantum spin ice," Phys. Rev. Lett. 122, 117201 (2019).

${ }^{35}$ R. Sibille, N. Gauthier, E. Lhotel, V. Porée, V. Pomjakushin, R. A. Ewings, T. G. Perring, J. Ollivier, A. Wildes, C. Ritter, et al., "A quantum liquid of magnetic octupoles on the pyrochlore lattice," Nat. Phys. 16, 546-552 (2020).

${ }^{36}$ A. Poole, A. S. Wills, and E. Lelièvre-Berna, "Magnetic ordering in theXYpyrochlore antiferromagnet $\mathrm{Er}_{2} \mathrm{Ti}_{2} \mathrm{O}_{7}$ : a spherical neutron polarimetry study," J. Phys. Condens. Matter 19, 452201 (2007)

${ }^{37}$ L. Pan, S. K. Kim, A. Ghosh, C. M. Morris, K. A. Ross, E. Kermarrec, B. D. Gaulin, S. Koohpayeh, O. Tchernyshyov, and N. Armitage, "Low- energy electrodynamics of novel spin excitations in the quantum spin ice $\mathrm{Yb}_{2} \mathrm{Ti}_{2} \mathrm{O}_{7}$," Nat. Commu. 5, 1-7 (2014).

${ }^{38}$ H. R. Molavian, M. J. Gingras, and B. Canals, "Dynamically induced frustration as a route to a quantum spin ice state in $\mathrm{Tb}_{2} \mathrm{Ti}_{2} \mathrm{O}_{7}$ via virtual crystal field excitations and quantum many-body effects," Phys. Rev. Lett. 98, 157204 (2007).

${ }^{39}$ J. Ruff, B. Gaulin, J. Castellan, K. Rule, J. Clancy, J. Rodriguez, and H. Dabkowska, "Structural fluctuations in the spin-liquid state of $\mathrm{Tb}_{2} \mathrm{Ti}_{2} \mathrm{O}_{7}$," Phys. Rev. Lett. 99, 237202 (2007).

${ }^{40}$ Y. Tokiwa, T. Yamashita, D. Terazawa, K. Kimura, Y. Kasahara, T. Onishi, Y. Kato, M. Halim, P. Gegenwart, T. Shibauchi, et al., "Discovery of emergent photon and monopoles in a quantum spin liquid," J. Phys. Soc. Japan 87, 064702 (2018).

${ }^{41}$ R. Sibille, N. Gauthier, H. Yan, M. C. Hatnean, J. Ollivier, B. Winn, U. Filges, G. Balakrishnan, M. Kenzelmann, N. Shannon, et al., "Experimental signatures of emergent quantum electrodynamics in $\operatorname{Pr}_{2} \mathrm{Hf}_{2} \mathrm{O}_{7}$," Nat. Phys. 14, 711-715 (2018).

${ }^{42} \mathrm{O}$. Benton, "Instabilities of a U(1) quantum spin liquid in disordered nonKramers pyrochlores," Phys. Rev. Lett. 121, 037203 (2018).

${ }^{43} \mathrm{G}$. Chen, "“Magnetic monopole" condensation of the pyrochlore ice $\mathrm{U}(1)$ quantum spin liquid: Application to $\mathrm{Pr}_{2} \mathrm{Ir}_{2} \mathrm{O}_{7}$ and $\mathrm{Yb}_{2} \mathrm{Ti}_{2} \mathrm{O}_{7}$," Phys. Rev. B 94, 205107 (2016)

${ }^{44}$ A. Scheie, J. Kindervater, S. Zhang, H. J. Changlani, G. Sala, G. Ehlers, A. Heinemann, G. S. Tucker, S. M. Koohpayeh, and C. Broholm, "Multiphase magnetism in $\mathrm{Yb}_{2} \mathrm{Ti}_{2} \mathrm{O}_{7}$," Proc. Natl. Acad. Sci. , 202008791 (2020)

${ }^{45}$ H. Takatsu, H. Kadowaki, T. J. Sato, J. W. Lynn, Y. Tabata, T. Yamazaki, and K. Matsuhira, "Quantum spin fluctuations in the spin-liquid state of $\mathrm{Tb}_{2} \mathrm{Ti}_{2} \mathrm{O}_{7}$, ,'J. Phys. Condens. Matter 24, 052201 (2011)

${ }^{46}$ H. R. Molavian and M. J. P. Gingras, "Proposal for a [111] magnetization plateau in the spin liquid state of $\mathrm{Tb}_{2} \mathrm{Ti}_{2} \mathrm{O}_{7}$," J. Phys. Condens. Matter 21, 172201 (2009)

${ }^{47}$ J. Nasu, M. Udagawa, and Y. Motome, "Vaporization of kitaev spin liquids," Phys. Rev. Lett. 113, 197205 (2014).

${ }^{48} \mathrm{C}$. Hickey and S. Trebst, "Emergence of a field-driven U(1) spin liquid in the Kitaev honeycomb model," Nat. Commu. 10, 1-10 (2019).

${ }^{49}$ H. Takagi, T. Takayama, G. Jackeli, G. Khaliullin, and S. E. Nagler, "Concept and realization of Kitaev quantum spin liquids," Nat. Rev. Phys. 1, 264-280 (2019).

${ }^{50}$ Y. Motome and J. Nasu, "Hunting majorana fermions in Kitaev magnets," J. Phys. Soc. Japan 89, 012002 (2020).

${ }^{51}$ L. Savary and L. Balents, "Disorder-induced quantum spin liquid in spin ice pyrochlores," Phys. Rev. Lett. 118, 087203 (2017).

${ }^{52}$ N. Martin, P. Bonville, E. Lhotel, S. Guitteny, A. Wildes, C. Decorse, M. C. Hatnean, G. Balakrishnan, I. Mirebeau, and S. Petit, "Disorder and quantum spin ice," Phys. Rev. X 7, 041028 (2017).

${ }^{53}$ J.-J. Wen, S. Koohpayeh, K. Ross, B. Trump, T. McQueen, K. Kimura, S. Nakatsuji, Y. Qiu, D. Pajerowski, J. Copley, et al., "Disordered route to the coulomb quantum spin liquid: random transverse fields on spin ice in $\mathrm{Pr}_{2} \mathrm{Zr}_{2} \mathrm{O}_{7}$," Phys. Rev. Lett. 118, 107206 (2017).

${ }^{54}$ D. Bowman, E. Cemal, T. Lehner, A. Wildes, L. Mangin-Thro, G. Nilsen, M. Gutmann, D. Voneshen, D. Prabhakaran, A. Boothroyd, et al., "Role of defects in determining the magnetic ground state of ytterbium titanate," Nat. Commu. 10, 1-8 (2019).

${ }^{55}$ S. Petit, E. Lhotel, S. Guitteny, O. Florea, J. Robert, P. Bonville, I. Mirebeau, J. Ollivier, H. Mutka, E. Ressouche, et al., "Antiferroquadrupolar correlations in the quantum spin ice candidate $\mathrm{Pr}_{2} \mathrm{Zr}_{2} \mathrm{O}_{7}$," Phys. Rev. B 94, 165153 (2016).

${ }^{56}$ G. van de Velde, B. Lippens, S. Korf, and J. Boeijsma, "Powder diffraction data for the imperfect pyrochlore terbium titanate, $\mathrm{Tb}_{2} \mathrm{Ti}_{2} \mathrm{O}_{7}$," Powder Diffr. 5, 229-231 (1990).

${ }^{57}$ R. S. Kumar, A. L. Cornelius, M. Somayazulu, D. Errandonea, M. F. Nicol, and J. Gardner, "High pressure structure of $\mathrm{Tb}_{2} \mathrm{Ti}_{2} \mathrm{O}_{7}$ pyrochlore at cryogenic temperatures," Phys. Status Solidi B 244, 266-269 (2007).

${ }^{58}$ P. R. Scott, A. Midgley, O. Musaev, D. Muthu, S. Singh, R. Suryanarayanan, A. Revcolevschi, A. Sood, and M. Kruger, "High-pressure synchrotron x-ray diffraction study of the pyrochlores: $\mathrm{Ho}_{2} \mathrm{Ti}_{2} \mathrm{O}_{7}, \mathrm{Y}_{2} \mathrm{Ti}_{2} \mathrm{O}_{7}$ and $\mathrm{Tb}_{2} \mathrm{Ti}_{2} \mathrm{O}_{7}$," High Press Res 31, 219-227 (2011).

${ }^{59}$ E. Kermarrec, D. Maharaj, J. Gaudet, K. Fritsch, D. Pomaranski, J. Kycia, Y. Qiu, J. Copley, M. Couchman, A. Morningstar, et al., "Gapped and gapless short-range-ordered magnetic states with $(1 / 2,1 / 2,1 / 2)$ wave vectors in 
the pyrochlore magnet $\mathrm{Tb}_{2+x} \mathrm{Ti}_{2-x} \mathrm{O}_{7+\delta}$," Phys. Rev. B 92, 245114 (2015).

${ }^{60} \mathrm{~K}$. Vlášková, B. Vondráčková, S. Daniš, and M. Klicpera, "High temperature study on $\mathrm{Er}_{2} \mathrm{Ti}_{2} \mathrm{O}_{7}$ single crystal," Acta Phys. Pol. 137 (2020).

${ }^{61}$ M. Ruminy, L. Bovo, E. Pomjakushina, M. Haas, U. Stuhr, A. Cervellino, R. Cava, M. Kenzelmann, and T. Fennell, "Sample independence of magnetoelastic excitations in the rare-earth pyrochlore $\mathrm{Tb}_{2} \mathrm{Ti}_{2} \mathrm{O}_{7}$," Physical Review B 93, 144407 (2016).

${ }^{62}$ Y. Yasui, M. Soda, S. Iikubo, M. Ito, M. Sato, N. Hamaguchi, T. Matsushita, N. Wada, T. Takeuchi, N. Aso, and K. Kakurai, "Ferromagnetic transition of pyrochlore compound $\mathrm{Yb}_{2} \mathrm{Ti}_{2} \mathrm{O}_{7}$," J. Phys. Soc. Japan 72, 3014-3015 (2003)

${ }^{63}$ A. Yaouanc, P. D. de Réotier, L. Keller, B. Roessli, and A. Forget, "A novel type of splayed ferromagnetic order observed in $\mathrm{Yb}_{2} \mathrm{Ti}_{2} \mathrm{O}_{7}$," J. Phys. Condens. Matter 28, 426002 (2016)

${ }^{64}$ X. Liu, S. Middey, Y. Cao, M. Kareev, and J. Chakhalian, "Geometrical lattice engineering of complex oxide heterostructures: a designer approach to emergent quantum states," MRS Commun. 6, 133-144 (2016).

${ }^{65}$ J. Chakhalian, X. Liu, and G. A. Fiete, "Strongly correlated and topological states in [111] grown transition metal oxide thin films and heterostructures," APL Mater. 8, 050904 (2020).

${ }^{66}$ X. Liu, S. Singh, B. J. Kirby, Z. Zhong, Y. Cao, B. Pal, M. Kareev, S. Middey, J. W. Freeland, P. Shafer, et al., "Emergent magnetic state in (111)oriented quasi-two-dimensional spinel oxides," Nano Lett. 19, 8381-8387 (2019).

${ }^{67}$ X. Liu, F. Wen, E. Karapetrova, J.-W. Kim, P. Ryan, J. Freeland, M. Terilli, T.-C. Wu, M. Kareev, and J. Chakhalian, "In-situ fabrication and transport properties of (111) $\mathrm{Y}_{2} \mathrm{Ir}_{2} \mathrm{O}_{7}$ epitaxial thin film," Appl. Phys. Lett. 117, 041903 (2020).

${ }^{68}$ X. Liu, T. Asaba, Q. Zhang, Y. Cao, B. Pal, S. Middey, P. Kumar, M. Kareev, L. Gu, D. Sarma, et al., "Quantum spin liquids by geometric lattice design," arXiv preprint arXiv:1911.00100 (2019).

${ }^{69}$ T. A. Bojesen and S. Onoda, "Quantum spin ice under a [111] magnetic field: from pyrochlore to kagome," Phys. Rev. Lett. 119, 227204 (2017).

${ }^{70} \mathrm{H}$. Kadowaki, H. Takatsu, and M. Wakita, "Dimensional change of the quadrupole order in pseudospin-1/2 pyrochlore magnets under magnetic field in the [111] direction," Phys. Rev. B 98, 144410 (2018).

${ }^{71}$ L. Bovo, C. M. Rouleau, D. Prabhakaran, and S. T. Bramwell, "Layer-bylayer epitaxial thin films of the pyrochlore $\mathrm{Tb}_{2} \mathrm{Ti}_{2} \mathrm{O}_{7}$," Nanotechnology 28 , 055708 (2016).

${ }^{72}$ L. Bovo, X. Moya, D. Prabhakaran, Y.-A. Soh, A. Boothroyd, N. Mathur, G. Aeppli, and S. Bramwell, "Restoration of the third law in spin ice thin films," Nat. Commun. 5, 1-8 (2014).

${ }^{73}$ M. Kareev, S. Prosandeev, B. Gray, J. Liu, P. Ryan, A. Kareev, E. Ju Moon, and J. Chakhalian, "Sub-monolayer nucleation and growth of complex oxides at high supersaturation and rapid flux modulation," J. Appl. Phys. 109, 114303 (2011).

${ }^{74}$ S. Kaya and E. Yilmaz, "Modifications of structural, chemical, and electrical characteristics of $\mathrm{Er}_{2} \mathrm{O}_{3} / \mathrm{Si}$ interface under Co-60 gamma irradiation," Nucl. Instrum. Methods Phys. Res., B 418, 74-79 (2018).

${ }^{75} \mathrm{H}$. Wang, M. Simmonds, and J. Rodenburg, "Manufacturing of YbAG coatings and crystallisation of the pure and $\mathrm{Li}_{2} \mathrm{O}$-doped $\mathrm{Yb}_{2} \mathrm{O}_{3}-\mathrm{Al}_{2} \mathrm{O}_{3}$ system by a modified sol-gel method," Mater. Chem. Phys. 77, 802-807 (2003).

${ }^{76}$ W. Cartas, R. Rai, A. Sathe, A. Schaefer, and J. F. Weaver, "Oxidation of a $\mathrm{Tb}_{2} \mathrm{O}_{3}$ (111) thin film on Pt (111) by gas-phase oxygen atoms," J. Phys.
Chem. C 118, 20916-20926 (2014).

${ }^{77}$ S. Shibagaki and K. Fukushima, "XPS analysis on $\mathrm{Nb}-\mathrm{SrTiO}_{3}$ thin films deposited with pulsed laser ablation technique," J. Eur. Ceram. Soc. 19, 1423-1426 (1999).

${ }^{78}$ F. Wen, Y. Cao, X. Liu, B. Pal, S. Middey, M. Kareev, and J. Chakhalian, "Evolution of ferromagnetism in two-dimensional electron gas of $\mathrm{LaTiO}_{3} / \mathrm{SrTiO}_{3}$," Appl. Phys. Lett. 112, 122405 (2018).

${ }^{79}$ N. Guerfi, O. Bourbia, and S. Achour, "Study of erbium oxidation by xps and ups," in Materials Science Forum, Vol. 480 (Trans Tech Publ, 2005) pp. 193-196.

${ }^{80} \mathrm{~S}$. Scalese, S. Mirabella, and A. Terrasi, "XPS and RBS investigations of Si-Er-O interactions on a Si (100)-2x1 surface," Appl. Surf. Sci. 220, 231237 (2003).

${ }^{81}$ T.-M. Pan and T.-Y. Yu, "Comparison of the structural properties and electrical characteristics of $\operatorname{Pr}_{2} \mathrm{O}_{3}, \mathrm{Nd}_{2} \mathrm{O}_{3}$ and $\mathrm{Er}_{2} \mathrm{O}_{3}$ charge trapping layer memories," Semicond. Sci. Technol. 24, 095022 (2009).

${ }^{82} \mathrm{H}$. Wang, M. Simmonds, and J. Rodenburg, "Manufacturing of YbAG coatings and crystallisation of the pure and $\mathrm{Li}_{2} \mathrm{O}$-doped $\mathrm{Yb}_{2} \mathrm{O}_{3}-\mathrm{Al}_{2} \mathrm{O}_{3}$ system by a modified sol-gel method," Mater. Chem. Phys. 77, 802-807 (2003).

${ }^{83}$ T.-M. Pan and W.-S. Huang, "Physical and electrical characteristics of a high-k $\mathrm{Yb}_{2} \mathrm{O}_{3}$ gate dielectric," Appl. Surf. Sci. 255, 4979-4982 (2009).

${ }^{84}$ M. M. Rahman, M. Alam, A. M. Asiri, and M. Islam, "Fabrication of selective chemical sensor with ternary $\mathrm{ZnO} / \mathrm{SnO}_{2} / \mathrm{Yb}_{2} \mathrm{O}_{3}$ nanoparticles," Talanta 170, 215-223 (2017).

${ }^{85}$ C. J. Lee, S. Vashishtha, A. Sayal, and J. F. Weaver, "Oxidation of a c- $\mathrm{Tb}_{2} \mathrm{O}_{3}$ (111) thin film by the sequential formation of stoichiometric phases," Surf. Sci. 694, 121555 (2020).

${ }^{86}$ W. Cartas, R. Rai, A. Sathe, A. Schaefer, and J. F. Weaver, "Oxidation of a $\mathrm{Tb}_{2} \mathrm{O}_{3}$ (111) thin film on Pt (111) by gas-phase oxygen atoms," J. Phys. Chem. C 118, 20916-20926 (2014).

${ }^{87}$ Q. Wang, A. Ghasemi, A. Scheie, and S. Koohpayeh, "Synthesis, crystal growth and characterization of the pyrochlore $\mathrm{Er}_{2} \mathrm{Ti}_{2} \mathrm{O}_{7}$," CrystEngComm 21, 703-709 (2019).

${ }^{88}$ K. Arpino, B. Trump, A. Scheie, T. McQueen, and S. Koohpayeh, "Impact of stoichiometry of $\mathrm{Yb}_{2} \mathrm{Ti}_{2} \mathrm{O}_{7}$ on its physical properties," Phys. Rev. B 95, 094407 (2017).

${ }^{89} \mathrm{~K}$. Rule and P. Bonville, "Tetragonal distortion in $\mathrm{Tb}_{2} \mathrm{Ti}_{2} \mathrm{O}_{7}$ seen by neutron scattering," in J. Phys. Conf. Ser., Vol. 145 (IOP Publishing, 2009) p. 012027.

${ }^{90}$ J. Gardner, B. Gaulin, and D. M. Paul, "Single crystal growth by the floating-zone method of a geometrically frustrated pyrochlore antiferromagnet, $\mathrm{Tb}_{2} \mathrm{Ti}_{2} \mathrm{O}_{7}$," J. Cryst. Growth 191, 740-745 (1998).

${ }^{91}$ L. Zhang, W. Zhang, J. Zhu, Q. Hao, C. Xu, X. Yang, L. Lu, and X. Wang, "Synthesis of $\mathrm{Er}_{2} \mathrm{Ti}_{2} \mathrm{O}_{7}$ nanocrystals and its electrochemical hydrogen storage behavior," J. Alloys Compd. 480, L45-L48 (2009).

${ }^{92}$ B. Cao, J. Wu, N. Yu, Z. Feng, and B. Dong, "Structure and upconversion luminescence properties of $\mathrm{Er}^{3+}-\mathrm{Mo}^{6+}$ codoped $\mathrm{Yb}_{2} \mathrm{Ti}_{2} \mathrm{O}_{7}$ films," Thin Solid Films 550, 495-498 (2014).

${ }^{93}$ K. Baroudi, B. D. Gaulin, S. H. Lapidus, J. Gaudet, and R. Cava, "Symmetry and light stuffing of $\mathrm{Ho}_{2} \mathrm{Ti}_{2} \mathrm{O}_{7}, \mathrm{Er}_{2} \mathrm{Ti}_{2} \mathrm{O}_{7}$, and $\mathrm{Yb}_{2} \mathrm{Ti}_{2} \mathrm{O}_{7}$ characterized by synchrotron x-ray diffraction,” Phys. Rev. B 92, 024110 (2015). 